\title{
Mass loss and evolution of hot massive stars
}

\author{
Jorick S. Vink ${ }^{1}$ \\ ${ }^{1}$ Armagh Observatory, College Hill, Armagh, BT61 9DG, Northern Ireland, United Kingdom \\ email: jsv@arm.ac.uk
}

\begin{abstract}
We discuss the role of mass loss for the evolution of the most massive stars, highlighting the role of the predicted bi-stability jump that might be relevant for the evolution of rotational velocities during or just after the main sequence. This mechanism is also proposed as an explanation for the mass-loss variations seen in the winds from Luminous Blue Variables (LBVs). These might be relevant for the quasi-sinusoidal modulations seen in a number of recent transitional supernovae (SNe), as well as for the double-throughed absorption profile recently discovered in the $\mathrm{H} \alpha$ line of SN 2005gj. Finally, we discuss the role of metallicity via the $Z$ dependent character of their winds, during both the initial and final (Wolf-Rayet) phases of evolution, with implications for the angular momentum evolution of the progenitor stars of long gamma-ray bursts (GRBs).
\end{abstract}

Keywords. Stars: winds, stars: mass loss, stars: supergiants, stars: rotation, stars: supernovae

\section{Introduction}

Mass loss has a major effect on the evolution of stars of all initial masses, however its effect is most prominent for the more massive stars due to their large luminosities - in close proximity to the Eddington limit. Mass loss is relevant both in terms of evolutionary pathways as well as the properties of the pre-supernova (SN) circumstellar environments. In this contribution, we first discuss mass-loss predictions that are relevant for predicting the forward evolution of massive stars. There are actually two aspects that need to be accounted for: (i) the loss of mass as winds "peel off" the star's outer layers (Conti 1976), but as massive stars start their evolution as rapid rotators, also (ii) the associated loss of angular momentum (e.g. Langer 1998).

Towards the end of the main sequence massive stars encounter the so-called bi-stability jump, for which we discuss the implications of the loss of angular momentum. For the final stages, the evolution of angular momentum is particularly relevant for our understanding of the long gamma-ray burst (GRB) phenomenon, as the popular collapsar model (MacFadyen \& Woosley 1999) requires the core of the progenitor star to be rapidly rotating before collapse (but see also Lee \& Ramirez-Ruiz 2006). A key parameter in the story is that of metallicity, due to the $Z$-dependence of radiation-driven winds during both the main sequence and evolved Luminous Blue Variable (LBV) and Wolf-Rayet (WR) phases of massive star evolution.

In the canonical scenario of massive stars, LBVs represent a transitional phase between the main sequence and the WR phase, however we also discuss the possibility for a new evolutionary paradigm in which the variable winds of LBVs might betray themselves as the direct progenitors of SNe.

\section{Mass loss predictions}

The evolution of a massive star, with $M>30 M_{\odot}$ is largely determined by the strength of their winds, which depends on the luminosity $(L)$, mass $(M)$, and metallicity $Z$. 


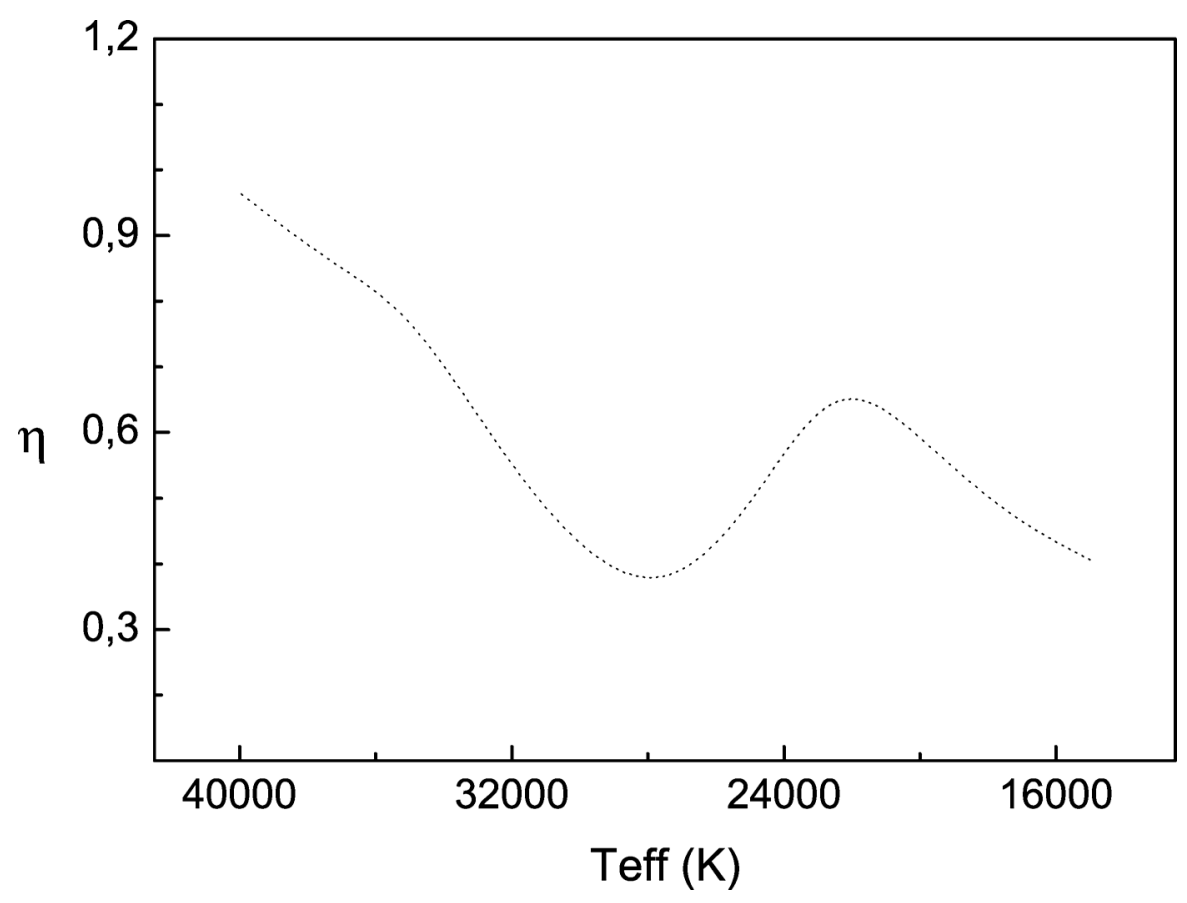

Figure 1. Predicted mass-loss behaviour as a function of effective temperature. Note the position of the local maximum at about $22000 \mathrm{~K}$, coincident with the position of the empirical bi-stability jump at spectral type B1 (Lamers et al. 1995).

The Fe-group elements are particularly efficient scatterers of photons at specific line frequencies responsible for the amount of mass loss, whilst the CNO elements set the wind terminal velocity (Vink et al. 1999). There are currently two basic methods in use for predicting the mass-loss rates from massive stars, which have been reviewed in detail by Vink (2006). In short, the first method concerns the modified-CAK (Castor et al. 1975) method (Kudritzki \& Puls 2000), the second one involves the Monte Carlo approach (Abbott \& Lucy 1985, Vink et al. 2000). Both methods have their pros and cons. In the first approach, the wind hydrodynamics are more or less self-consistently solved for (albeit using depth-dependent force multiplier parameters), however multi-line scattering is not accounted for. This aspect is included in the second approach, where the line acceleration is calculated for all radii, although most Monte Carlo predictions do not properly account for the wind hydrodynamics (but see Vink et al. 1999).

Vink et al. $(1999,2000)$ predicted the mass-loss rates of OB supergiants as a function of the stellar parameters $\left(L, M\right.$, and $\left.T_{\text {eff }}\right)$ including multiple scatterings on line and continuum opacity with a Monte Carlo approach and their mass-loss rates were computed as a function of the wind terminal velocity - a parameter accurately (within 10\%) retrievable from ultraviolet P Cygni line profiles. This is in stark contrast to empirical mass-loss rates which are highly uncertain (by factors up to 3-10) due to uncertainties in wind ionization and wind inhomogeneities (see Hamann et al. 2008 for a recent overview on the issue of wind clumping). The Vink et al. mass-loss rates are found to scale as:

$$
\dot{M} \propto L^{2.2} M^{-1.3} T_{\text {eff }}^{1}\left(v_{\infty} / v_{\text {esc }}\right)^{-1.3}
$$

showing that $\dot{M}$ depends rather steeply on the stellar luminosity $\left(L^{2.2}\right)$. The reason for this is that brighter stars have denser winds and Monte Carlo predictions yield an 
increasingly larger mass-loss rate than modified-CAK predictions. The Vink et al. massloss recipes are widely used in models for massive star evolution (e.g. Meynet \& Maeder 2003, Limongi \& Chieffi 2006, Eldridge \& Vink 2006, Brott et al. in prep.).

Figure 1 depicts how the predicted mass-loss rates vary as a function of $T_{\text {eff }}$ and thus how a massive star may find its wind change during its coarse of evolution. The predictions are expressed in terms of the wind momentum efficiency, or wind performance number, $\frac{\dot{M} v_{\infty}}{L / c}$. The figure shows a declining wind efficiency with $T_{\text {eff }}$. At high temperatures $(\sim 40000 \mathrm{~K})$ the wind momentum is large due to the fact that the radiative flux and the opacity have a good "match" with respect to their wavelength distribution. However, when $T_{\text {eff }}$ drops, the stellar flux moves away from its maximum towards lower (optical) wavelengths, which results in an ever-growing mismatch between the flux and the ultraviolet line opacity. At $\sim 25000 \mathrm{~K}$, a sudden mass-loss discontinuity is noted. This is due to an increased Fe opacity when Fe IV recombines, and the more abundant Fe III lines provide most of the line force in the inner wind (Vink et al. 1999). This "bi-stability jump" (Pauldrach \& Puls 1990) may recently have been confirmed in radio data that appear to confirm the presence of a local maximum (Benaglia et al. 2007, Markova \& Puls 2008), however it should also be noted that the predicted values below the temperature of the jump appear to be much larger than those found from empirical modelling by up to a factor of 10 (Vink et al. 2000, Trundle \& Lennon 2005, Crowther et al. 2006).

The bi-stability jump where winds change from a low $\dot{M}$, fast wind, to a high $\dot{M}$, slow wind may comprise an important ingredient for stellar evolution calculations when stars evolve off their main-sequence positions towards the lower $T_{\text {eff }}$ part of the HertzsprungRussell diagram (HRD). This is not only relevant for their mass loss, but also for the associated loss of angular momentum.

\section{Angular momentum loss}

Massive stars rotate rapidly at birth (with $v_{\text {rot }} \simeq 200-300 \mathrm{~km} \mathrm{~s}^{-1}$ ) and remain relatively rapid rotators throughout their main-sequence lifetimes. Obviously, $v_{\text {rot }}$ decreases due to the angular momentum loss via stellar winds, which implies that the effects are largest at the highest initial masses and luminosities, and metallicities. Furthermore, when the objects evolve off the main sequence, they swell up to become (super)giants, and $v_{\text {rot }}$ is anticipated to drop due to the increase in stellar radius (e.g. Hunter et al. 2008). However, is this the entire story or is the bi-stability jump also of relevance?

Figure 2 shows a recent figure from Markova \& Puls (2008) showing how the rotational velocity of Galactic OB supergiants depends on spectral type. It can be noted that $v \sin i$ drops from $\sim 100 \mathrm{~km} \mathrm{~s}^{-1}$ to $\sim 50 \mathrm{~km} \mathrm{~s}^{-1}$ close to spectral type B1 - the position of the bi-stability jump (Lamers et al. 1995, Crowther et al. 2006). As we are interested in checking whether the predicted jump in mass loss by a factor of five at the bi-stability jump (Vink et al. 1999, 2000) might potentially explain the steep drop in rotation due to the loss of angular momentum evolutionary tracks were computed with this in mind (Brott et al. in prep.). Figure 3 shows both the Vink et al. (2000) mass-loss rates (dotted line) and the predicted rotational velocity (solid line) of a Galactic $40 M_{\odot}$ star which had a initial rotational velocity of $265 \mathrm{~km} \mathrm{~s}^{-1}$ on the zero-age main-sequence (ZAMS). In order for the angular momentum removal to be maximal, a rather large overshooting parameter of 0.335 of a pressure scale-height was employed, as this provides a long time interval on the MS for the mass loss to be most efficient. 


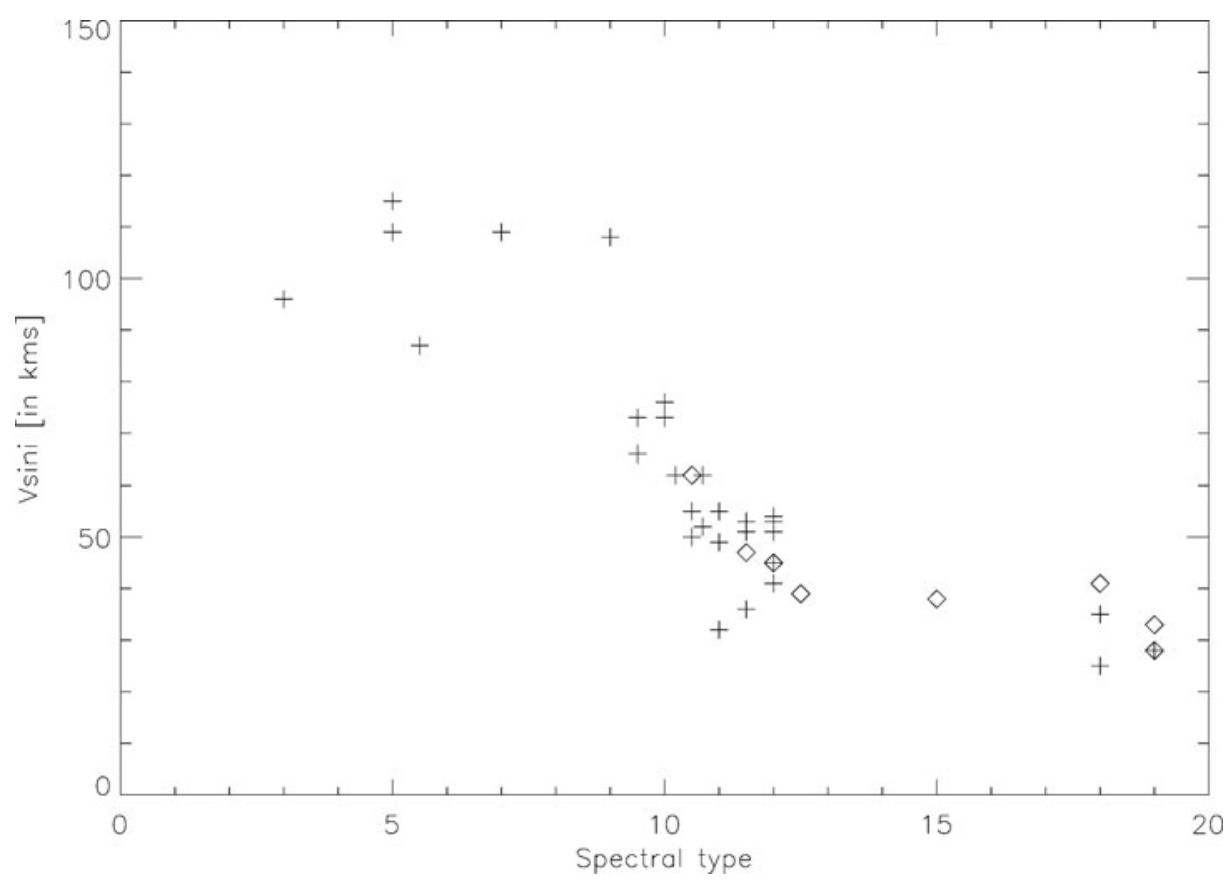

Figure 2. The projected rotational velocity, $v \sin i$ of Galactic B supergiants as a function of spectral type, starting with O3 to O9 and then switching to B0 (at 10) and to A0 (at 20). It can be noted that $v \sin i$ drops from $\sim 100 \mathrm{~km} \mathrm{~s}^{-1}$ to $\sim 50 \mathrm{~km} \mathrm{~s}^{-1}$ at around spectral type B0, which is close to the spectral type of the bi-stability jump B1 (Lamers et al. 1995). The figure has been adapted from Markova \& Puls (2008).

As an aside, we note that the drop in rotational velocities at a specific temperature is reminiscent of a situation encountered for Horizontal Branch stars (e.g. Behr et al. 2000). Vink (2007a) recently reviewed whether the steep jump at $10000 \mathrm{~K}$ could be due to the onset of radiation-driven winds as a result of the higher metal content for objects warmer than the jump temperature.

\section{Could the changing winds of Luminous Blue Variables change the evolutionary paradigm?}

Above we considered the effects of the bi-stability jump for main-sequence stars evolving from hot to cool temperatures. However, even more dramatic effects might occur for objects that have already lost a large fraction of their initial mass, finding themselves in close proximity to the Eddington limit. These Luminous Blue Variables change their effective temperatures on a variety of timescales (Humphreys \& Davidson 1994, Vink 2008). The micro variations are not noticably different from small-amplitude variations in other BA supergiants. At the other extreme, we find the super-outburst of objects such as Eta Car, sometimes referred to as SN-impostors, when observed in other galaxies (Van Dyk et al. 2000, Maund et al. 2006). We note that only two of these super-outbursts have been identified in the Milky Way (the eruptions of Eta Car in the 19th and P Cyg in the 17th century). The mass-loss rates involved in these giant eruptions are of order $0.1 M_{\odot} \mathrm{yr}^{-1}$ and are too large to be explained by line acceleration. However, continuumdriven winds may well be able to provide the necessary driving (Smith \& Owocki 2006). Most typifying for the class are the S Dor variations, where objects vary on timescales 
Milkyway: 40 Msun, 265 km/s @ ZAMS

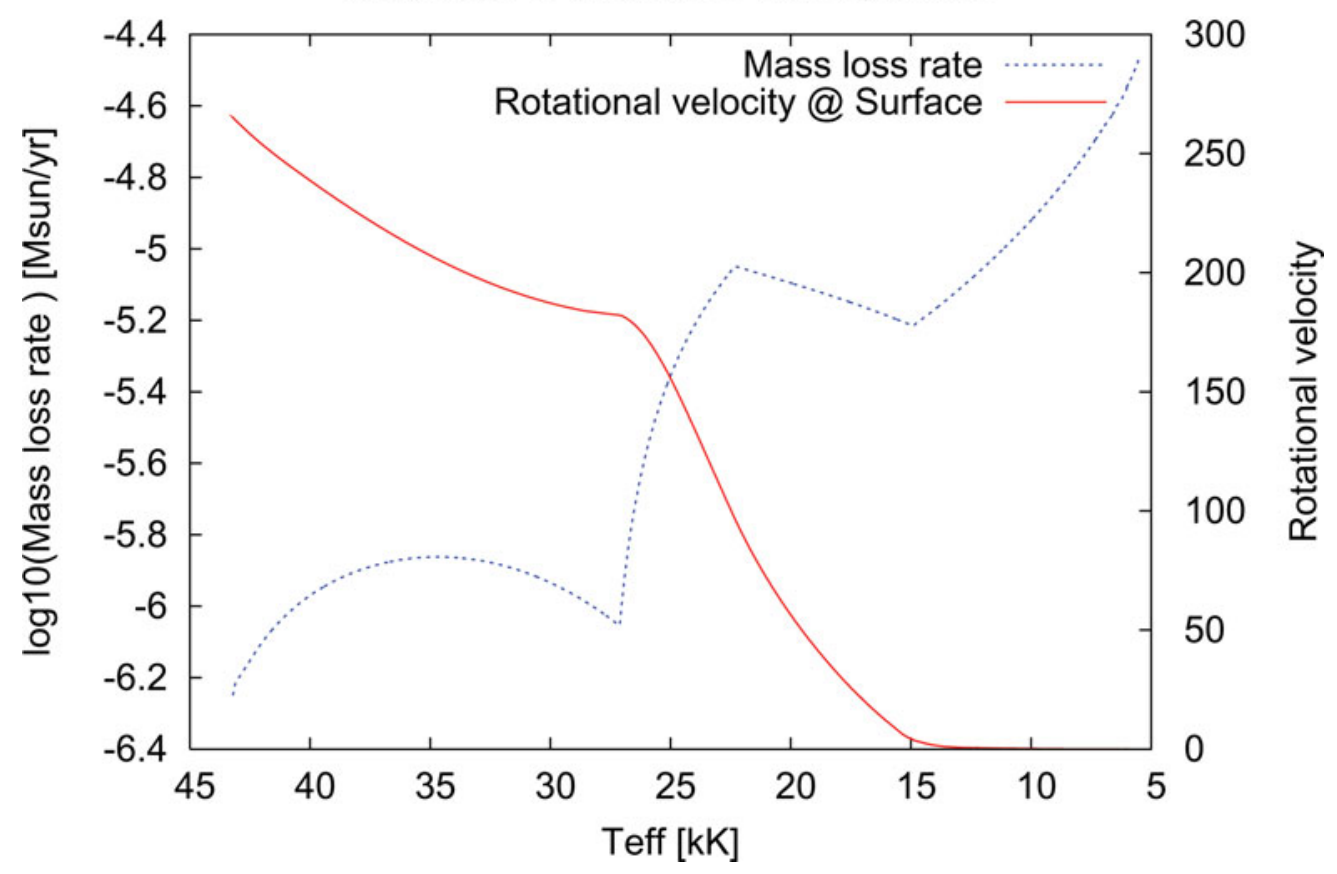

Figure 3. Mass-loss rate (dotted line) and rotational velocity (solid line) of a Galactic $40 M_{\odot}$ star which had a initial rotational velocity of $265 \mathrm{~km} \mathrm{~s}^{-1}$ on the ZAMS. A rather large overshootig parameter of 0.335 of a pressure scale-height was employed. The mixing efficiency of $f c=0.0228$ represents an efficiency factor with which the diffusion coefficients of the different rotational mixing processes are multiplied (see Hunter et al. 2008, Brott et al., in prep. for further details.) Note that the second mass-loss increase below $15000 \mathrm{~K}$ is due to a swith from the Vink et al. (2000) recipe to a calibration by Nieuwenhuizen \& de Jager (1990) - consistent with the position of the second bistability jump predicted by Vink et al. and observed by Lamers et al. (1995) around spectral type A0.

of years to decades. When LBVs such as AG Car - one of the prototypes - change their radii on their S Dor timescales, they show large mass-loss variations (Stahl et al. 2001). Such variable wind behaviour has qualitatively been reproduced by radiation-driven wind models of Vink \& de Koter (2002). We anticipate that this type of wind behaviour may result in a circumstellar medium consisting of concentric shells with varying densities, which may have ramifications for the end-points of massive stars. Kotak \& Vink (2006) suggested that the quasi-periodic modulations seen in the radio lightcurves of some supernovae (SNe), such as 2001ig (Ryder et al. 2004) and 2003bg (Soderberg et al. 2006) may indicate that LBVs could be the direct progenitors of some SNe.

At first this seems to contradict stellar evolution calculations, which do not predict LBVs to explode, and such a scenario was until recently considered "wildly speculative" (Smith \& Owocki 2006). However, the intruiging supernova 2006jc showed a giant eruption just 2 years prior to explosion (Pastorello et al. 2007, Foley et al. 2007) which may add some confidence to the Kotak \& Vink suggestion that LBVs might explode. There have been a number of other studies suggestting that LBVs may explode. Gal Yam et al. (2007) reported the detection of a most luminous progenitor of SN 2005gl. Although the properties of the potential progenitor star are consistent with that of an LBV, a hypergiant cannot be classified as an LBV until it has shown S Dor or Eta-Car-type variability (Humphreys \& Davidson 1994, Vink 2008). Another interesting hint that LBVs 
may explode arises from the similarities in LBV nebula morphologies and the circumstellar medium of SN 1987A (Smith 2007). Finally, one the most luminous supernova ever recorded, SN 2006gy (e.g. Ofek et al. 2007) may also have been an Eta Carinae type LBV (Smith et al. 2007).

As current state-of-the-art stellar evolution calculations do not predict LBVs to explode, this represents a major unresolved problem in the physics of massive stars. In the generally upheld picture for the evolution of the most massive stars, LBVs are considered "transitional" objects in a phase before entering the He-burning WR stage, by the end of which the WR star is expected to explode as a type Ib/c supernova. The reason for the common (Conti 1976) scenario:

$$
O \rightarrow \mathrm{LBV} \rightarrow \mathrm{WR} \rightarrow \mathrm{SN}
$$

is that LBVs are He (and N) rich compared to O stars, yet H-rich (thus He-poor) compared to WR stars. The situation is even more complex as there is also a group of high-luminosity late-type WR stars which are H-rich and seem closely related to the classical LBVs in quiescence. In particular, we note that the R127 was a late-type WN star (Of/WN9), before it went into outburst. Nonetheless, the picture of a relatively short-lived, some $10^{4} \mathrm{yrs}$, core H-burning LBV phase prior to a more extensive spell of a few times $10^{5}$ yrs core He-burning WR phase seemed well established - until recently.

Whilst the quasi-sinusoidal modulations in the radio lightcurves of transitional $\mathrm{SNe}$ may possibly also be explained by alternative scenariost, it might be relevant that the same underlying mechanism, i.e. wind bi-stability, might account for wind-velocity variations seen spectroscopically in the SN SN 2005gj (Trundle et al. 2008). Here, the variable winds are inferred from double P Cygni components (see Fig. 4) which appear almost identical to those seen in the $\mathrm{H} \alpha$ profiles of the well-known S Dor variables AG Car and HD 160529. It should also be noted that the timescales and the spectroscopically measured wind velocities of SN 2005gj, with $v_{\infty} \simeq 100-200 \mathrm{~km} \mathrm{~s}^{-1}$, are consistent with those of LBVs, whilst they are yet again not consistent with those of the much slower RSG winds $\left(\sim 10 \mathrm{~km} \mathrm{~s}^{-1}\right)$, or the much faster WR winds $\left(\simeq 1000-5000 \mathrm{~km} \mathrm{~s}^{-1}\right)$.

\section{Mass loss as a function of metallicity}

Metallicity is a key parameter in the physics of stars and star-forming galaxies, largely via metallicity-dependent stellar winds. We compare the predictions of O star mass-loss rates (Vink et al. 2001) with recent empirical mass-loss rates from Mokiem et al. (2007) using the so-called wind momentum-luminosity relationship (Kudritzki \& Puls 2000) for Galactic, Large Magallanic Cloud, and Small Magallanic Cloud O stars in the $Z$ range

$\dagger$ Although there are other explanations for these radio modulations, none of these are entirely satisfactory. Ryder et al. (2004) suggested the modulations might be due to a WR pinwheel system where a secondary star perturbs the circumstellar medium of the primary WR star. Although this remains possible (though the inferred radial spacings in Ryder et al. (2004) are incorrect by a factor of 10 , see Kotak \& Vink 2006), the fact that SN 2003bg is so similar to SN 2001ig led Soderberg et al. (2006) to suggest the modulations are more likely due to a variable WR wind of a single star, resulting in concentric shells. However, such variability has never been observed in WR stars. This shortcoming was alleviated with the S Dor LBV suggestion of Kotak \& Vink. Finally, we mention the possibility that the radio modulations might be due to a variable wind of a pulsating red supergiant (RSG; Heger et al. 1997), however the problem with such a scenario is that a RSG is H-rich, whilst SN 2003bg was first classified as a SN Ic. The fact that SNe 2001ig and 2003bg showed exactly the opposite transitional behaviour between type I and II, or H-rich or H-poor, was an extra reason for Kotak \& Vink to consider LBVs as possible progenitors, as LBVs are H-rich compared to WR stars, but H-poor compared to RSGs. 


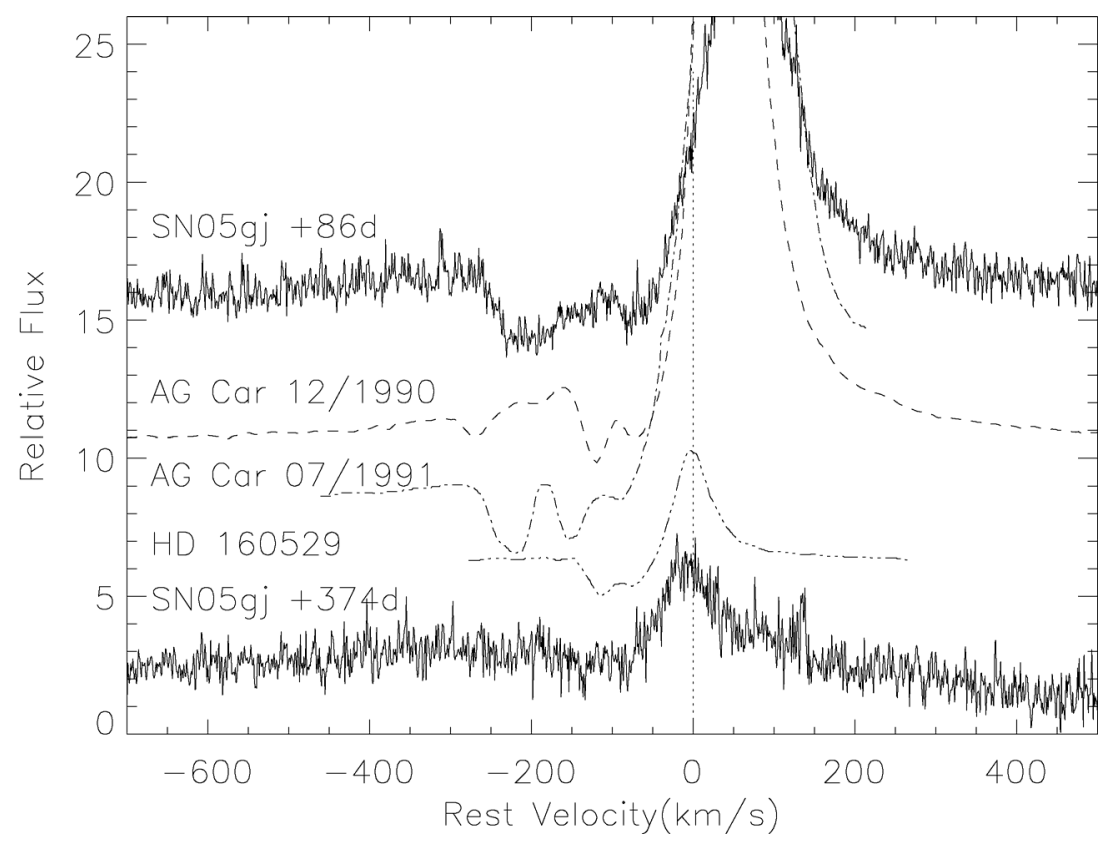

Figure 4. Multiple absorption components seen in the P Cygni H $\alpha$ profile of SN 2005gj (top) in comparison to the LBVs AG Car and HD 160529. The figure has been taken from Trundle et al. (2008).

from solar to only $20 \%$ solar. We note that for all three galaxies, the empirical rates are somewhat larger (by a factor $\sim 2$ ) than predicted by Vink et al. (see Fig.5). As the empirical rates are most likely affected by wind clumping (e.g. Bouret et al. 2003, Martins et al. 2005, Hamann et al. 2008), the empirical rates are likely maximal. Therefore, when we assume a modest clumping factor corresponding to an empirical $\dot{M}$ reduction of a factor $\sim 2$, the empirical rates show very good agreement with theory. The wind clumping factor however remains an unsolved problem in stellar astrophysics and if the true wind clumping is larger than assumed, with empirical $\dot{M}$ overestimates of $\sim 10$ as some studies suggest (e.g. Fullerton et al. 2006), the current mass-loss predictions might also be too large. This is certainly an important topic for future investigation.

Massive stars lose mass at even higher rates during the more evolved LBV and WR phases. During the latter phase, the outer layers become strongly chemically enriched, which may potentially modify mass loss through winds. Vink \& de Koter (2005) investigated the mass loss versus $Z$ dependence for late-type WR stars using a Monte Carlo approach (see Fig. 6). Despite the overwhelming presence of carbon at all $Z, \dot{M}$ does not show a $Z$-independent behaviour (as was generally assumed previously), but WR mass loss depends strongly on the iron (Fe) opacity, just like for O stars (Vink et al. 1999, Gräfener \& Hamann 2008).

Furthermore, although the $\dot{M}$ versus $Z$ dependence is consistent with a power-law decline in the observable Universe down to $\log Z / Z_{\odot} \sim-3$, it flattens off for extremely low $Z$ models. The reason is that carbon, nitrogen, oxygen, hydrogen and helium take over the driving from Fe which dominates the higher $Z$ domain. The strong $Z$-dependence of WR winds where the WR $\dot{M}$ drops by orders of magnitude, might represent a key result for the high incidence of long-duration GRBs at low metallicity. The favoured progenitors of long GRBs are thought to be rapidly rotating WR stars. However, most Galactic WR 


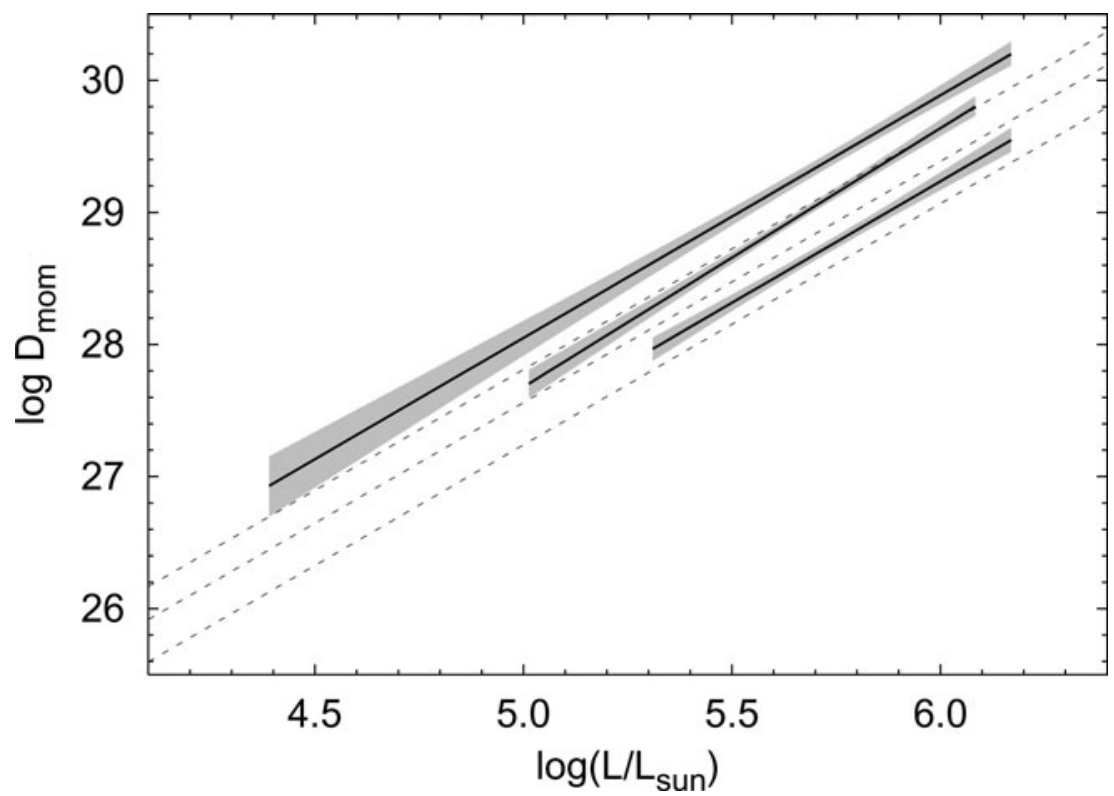

Figure 5. This figure represents a confrontation between $\mathrm{O}$ star mass-loss predictions (dashed line) and recent empirical mass-loss rates in the form of the so-called wind momentum-luminosity relationship for Galactic (top), LMC (middle) and SMC (bottom) O stars. The figure is from Mokiem et al. (2007).

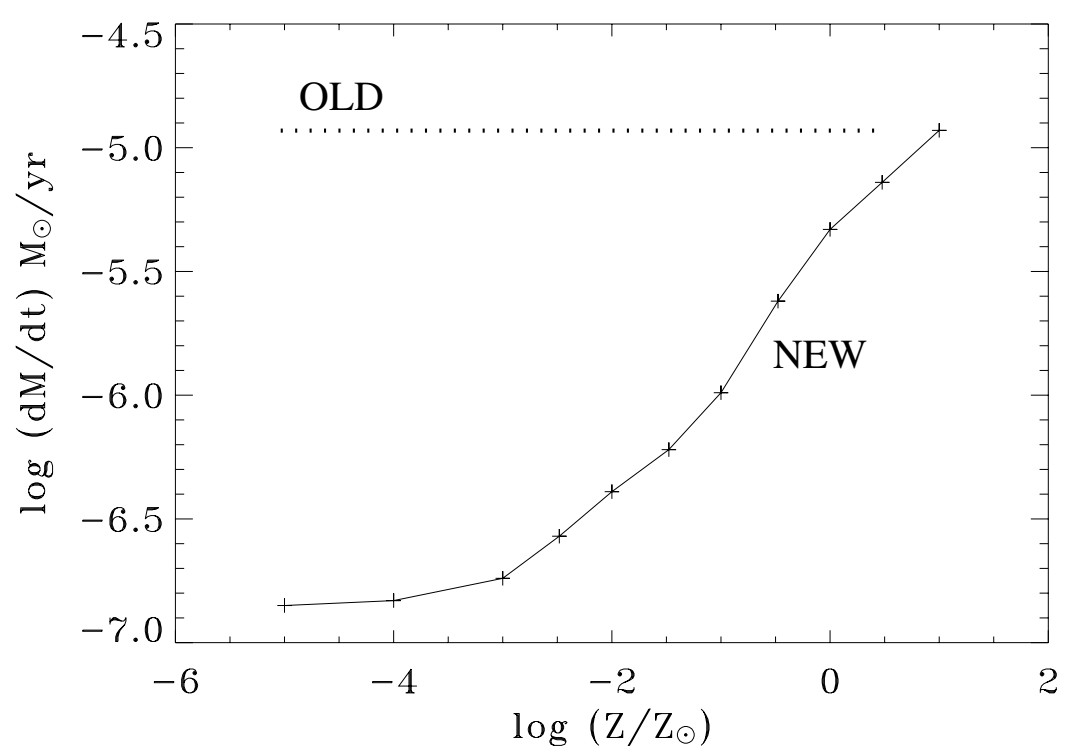

Figure 6. Z-dependent mass-loss predictions for Wolf-Rayet stars (Vink \& de Koter 2005). Albeit the overwhelming presence of carbon for all $Z$, the new WR mass-loss rate does not show $Z$-independent behaviour as assumed previously (dotted line). The new computations show that WR mass loss depends strongly on iron $(Z)$ - a key result for predicting a high occurrence of long-duration GRBs at low $Z$. 
stars are slow rotators, as stellar winds probably remove the necessary stellar angular momentum, potentially posing a challenge to the collapsar model for GRBs.

Observational data however indicate that GRBs occur predominately in low metallicity (Z) galaxies (e.g. Le Floc'h et al. 2003, Prochaska et al. 2004, Vreeswijk et al. 2004, Modjaz et al. 2008), which may resolve the problem: lower $Z$ leads to less mass loss, which may inhibit angular momentum removal, allowing WR stars to remain rotating rapidly until collapse (Yoon \& Langer 2005, Woosley \& Heger 2006). As a test of this scenario, Vink (2007b) performed a linear spectropolarimetry survey of WR stars in the low $Z$ environment of the LMC and found an incidence of line polarisation effects in LMC WR stars as low as that of the Galactic sample of Harries et al. (1998). This suggests that the threshold metallicity where significant differences in WR rotational properties occur is below that of the LMC (at $Z \sim 0.4 Z_{\odot}$ ), possibly constraining GRB progenitor channels to this upper metallicity.

\section{Conclusions}

We presented theoretical mass-loss rates and their implications for the peeling off and angular momentum loss of massive stars during the various evolutionary stages from the main sequence, to the LBV, and WR phases. The role of the bi-stability jump at an effective temperature of $\sim 25000 \mathrm{~K}$ was discussed in the context of the observed drop in rotational velocities in this part of the Hertzsprung-Russell diagram as well as for the variable winds of LBVs, suggesting these objects could be in a direct pre-SN state of massive star evolution.

\section{Acknowledgements}

We would like to thank all our collegues and friends in the field of massive stars and in particular to Alex de Koter and Rubina Kotak. Special thanks also to Ines Brott and Norbert Langer for computing the evolution of the rotation rate with time and temperature.

\section{References}

Abbott, D. C. \& Lucy, L. B. 1985, ApJ 288, 679

Benaglia, P., Vink, J. S., Marti, J., Maiz Apellaniz, J., Koribalski, B. \& Crowther, P. A. 2007, A\&A 467, 1265

Behr, B. B., Cohen, J. G., \& McCarthy, J. K. 2000, ApJ 531, L37

Bouret, S.-C., Lanz, T. \& Hillier, D. J. 2003, ApJ 595, 1182

Castor, J., Abbott, D. C. \& Klein, R. I. 1975, ApJ 195, 157

Conti, P. S. 1976, MSRSL 9, 193

Crowther, P. A., Lennon, D. J. \& Walborn, N. R. 2006, A\&A, 446, 279

Eldridge, J. J. \& Vink, J. S. 2006, A\&A, 452, 295

Foley, R. J., Smith, N., Ganeshalingam, M., et al. 2007, ApJ 657, L105

Fullerton, A. W., Massa, D. L. \& Prinja, R. K. 2006, ApJ 637, 1025

Gal-Yam, A., Leonard, D. C., Fox, D. B., et al. 2007,, ApJ 656, 372

Gräfener, G. \& Hamann, W.-R. 2008, A\&A 482, 945

Hamann, W.-R., Oskinova, L. M., \& Feldmeier, A. 2008, Clumping in Hot-Star Winds

Harries, T. J., Hillier, D. J., \& Howarth, I. D. 1998, MNRAS 296, 1072

Heger, A., Jeannin, L., Langer, N., \& Baraffe, I. 1997, A\&A 327, 224

Humphreys, R. M. \& Davidson, K. 1994, PASP 106, 1025

Hunter, I., Lennon, D. J., Dufton, P. L., Trundle, C., Simon-Diaz, Smartt, S. J., Ryans, R. S. I., \& Evans, C. J. 2008, A\&A 479, 541

Kotak, R. \& Vink, J. S. 2006, A\&A 460L, 5 
Kudritzki, R. P., Puls, J. 2000, ARA\&A 38, 613

Lamers, H. J. G. L. M., Snow, T. P., \& Lindholm, D. M. 1995, ApJ 455, L269

Langer N., 1998, A\&A 329, 551

Lee, W. H. \& Ramirez-Ruiz, E. 2006, ApJ 641, 961

Le Floc'h, E., Duc, P.-A., Mirabel, I. F., et al. 2003, A\&A 400, 499

Limongi, M., Chieffi, A. 2006, ApJ 647, 483

MacFadyen, A. I. \& Woosley, S. E. 1999, ApJ 524, 262

Martins, F., Schaerer, D., Hillier, D. J., et al. 2005, A\&A 441, 735

Markova, N. \& Puls, J. 2008, A\&A 478, 823

Maund, J. R., Smartt, S. J., Kudritzki, R.-P, et al. 2006, MNRAS 369, 390

Meynet, G. \& Maeder, A. 2003, A\&A 404, 975

Modjaz, M., Kewley, L., Kirshner, R. P., et al. 2008, AJ 135, 1136

Mokiem, M. R., de Koter, A., Vink, J. S. 2007, et al., A\&A 473, 603

Nieuwenhuijzen, H. \& de Jager, C. 1990, A\&A 231, 134

Ofek, E. O., Cameron, P. B., Kasliwal, M. M., et al. 2007, ApJ 662, 1129

Pastorello, A., Smartt, S. J., Mattila,S., et al. 2007, MNRAS 377, 1531

Pauldrach, A. W. A. \& Puls, J. 1990, A\&A 237, 409

Prochaska, J. X., Bloom, J. S., Chen, H.-W., et al. 2004, ApJ 611, 200

Ryder, S. D., Sadler, E. M., Subrahmanyan, R., et al. 2004, MNRAS 349, 1093

Smith, N. 2007, AJ 133, 1034

Smith, N. \& Owocki, S. P. 2006, ApJ 645, 45

Smith, N., Li, W., Foley, R.J, \& et al. 2007b, ApJ, 666, 1116

Soderberg, A. M., Chevalier, R. A., Kulkarni, S. R., \& Frail, D. A. 2006, ApJ 651, 1005

Stahl, O., Jankovics, I., Kovacs, J., et al. 2001, A\&A 375, 54

Trundle, C. \& Lennon, D. J. 2005, A\&A 434, 677

Trundle, C., Kotak, R., Vink, J. S., \& Meikle, W. P. S. 2008, A\&A 483, 47

Van Dyk, S. D., Peng, C. Y., King, J. Y., et al. 2000, PASP 112, 1532

Vink, J. S. 2006, ASPC 353, 113 (astro-ph/0511048)

Vink, J. S. 2007a, in: "Unsolved Problems in Stellar Physics: A Conference in Honor of Douglas Gough, AIPC 948, 389

Vink, J. S. 2007b, A\&A 469, 707

Vink, J. S. 2008, in: "Eta Carinae", eds R.M. Humphreys \& K. Davidson, Springer

Vink, J. S. \& de Koter, A. 2002, A\&A 393, 543

Vink, J. S. \& de Koter, A. 2005, A\&A 442587

Vink, J. S., de Koter, A., \& Lamers, H. J. G. L. M. 1999, A\&A 345, 109

Vink, J. S., de Koter, A., \& Lamers, H. J. G. L. M. 2000, A\&A 362, 295

Vink, J. S., de Koter, A., \& Lamers, H. J. G. L. M. 2001, A\&A 369, 574

Vreeswijk, P. M., Ellison, S. L., Ledoux, C., et al. 2004, A\&A 419, 927

Woosley, S. E. \& Heger, A. 2006, ApJ 637, 914

Yoon, S.-C. \& Langer, N. 2005, A\&A, 443, 643

\section{Discussion}

TURCK-CHIEzE: What are the limitations of the mass loss calculation as far as the opacities are concerned: do you use detailed spectra? do you introduce radiative acceleration from theoretical estimates, is the knowledge of these quantities determinant in the calculation?

VINK: As far as the opacities are concerned: we include all elements from H-Zn (1-30) in our line list in Monte Carlo. Before we compute models at zero metallicity, we need a more comprehensive dynamical framework. We have successfully solved the wind dynamics for a few cases, but we want to be able to provide this over the entire HRD. 
MEYNET: The argument for a $\mathrm{Z}$ dependance of $\dot{\mathrm{M}}$ during the WR phase is quite convincing and I agree with you that it may have an impact on the way the WC to WN ratio varies as a function of $\mathrm{Z}$. But when comparing WR populations with models you should not only compare the way the $\mathrm{WN} / \mathrm{WC}$ ratio varies with $\mathrm{Z}$ but also show the $\mathrm{WR} / \mathrm{O}$ number ratio varies with metallicity.

VINK: I agree with you. The point is that there are many physical mechanisms (rotation, B field, mass loss, binarity) responsible for how an O star becomes WR. To make comprehensive stellar and population synthesis models all these should of course be included. But I think we are still in a phase where we need to identify the relevant physical processes and test these for "simple" cases. To explain the drop in WC to WN, the mass loss versus Fe metallicity dependence may well be the first order effect. That was the point of that study.

Belczynski: This is a comment on G. Meynet question $\rightarrow$ that the wind mass loss rates predicted in your simulations may reproduce/give metallicity dependence for W-R stars, but they will significantly underproduce the number of W-R stars. That problem/issue can be avoided if one takes into account (quite easy) $\rightarrow$ formation of W-R stars in binaries (envelope removal in RLOF): in fact most massive stars are in close binaries and are subject to RLOF.

VINK: Again the point was not how to make WR stars. There are several routes, mass loss, quasi-homogeneous evolution, and, indeed, RLOF in binaries. So to get the complete picture you need to consider all these possibilities for a range of masses, metallicities, binary parameters, $v \sin i$, etc. Do you include mixing? B-field? etc. I do not think theory is ready for that. Does not mean we should not try!

PoDsiadlowski: I think that one has to be careful of what one calls an LBV. Some people call everything that shows variable mass loss an LBV. I do not think that this is very helpful. There are other physical mechanisms that causes eruptive events, e.g, binary mergers. These are theoretically predicted to be quite common and transient events in the near future are expected to detect these.

VINK: I do not want to exclude the possibility of binary mergers, they may even be quite common in dense systems. I am interested in your predictions and numbers as to how common they are. I do want to say though find there is evidence for blue supergiants with variable $\mathrm{T}_{\text {eff }}$ and variable winds and some of these have shown giant $\eta$ Car eruptions, for instance P Cygni in 1600. 